\title{
Method of cumulative particle for experimental study of multibaryon production
}

\author{
Boris Kostenko* \\ JINR, Dubna, Russia \\ E-mail: bkostenko@jinr.ru \\ Jan Pribiš \\ Technical University, Košice, Slovakia \\ E-mail: jan.pribis@tuke.sk \\ Valentina Filinova \\ JINR, Dubna, Russia \\ E-mail: filinovaejinr.ru
}

\begin{abstract}
After collisions with impinging particle, a density fluctuation of nuclear matter may turn into a multibaryon (MB) with chiral symmetry restored in its interior. This method of observation of the chiral phase transition has its advantages due to a relatively moderate number of secondary particles to be measured. We suggest to use a cumulative particle as a trigger for a detection of MB decay products. Estimations show that its appearance is a signature of "deep cooling" of MB, which brings it close to the unexcited state. This gives a chance to separate MB from the secondary particle background. For separation of events with MB production, a role of intranuclear collisions of MB decay products should also be evaluated. We study experimental data taken with the EVA spectrometer at BNL as a simplified form of the problem. Analysis of intranuclear interactions before and after hard scattering of protons from SRC leads to a plausible conclusion that there were $\pi$-mesons missed in the experiment. Other logically permissible possibilities such as a color anti-transparency effect and a presence of states reminding a fusiform (ellipsoidal) body in the wave function of ${ }^{12} \mathrm{C}$ are briefly discussed too. Then we show that the short range correlation mechanism is not the only reasonable explanation of the experimental data. Interpretations based on dibaryons pre-existent in nuclei before its interaction with the projectile, or created during this interaction are also possible. Some examples of detection of MB production by the cumulative particle method are given with estimations of their feasibility.
\end{abstract}

XXI International Baldin Seminar on High Energy Physics Problems September 10-15, 2012

JINR, Dubna, Russia

${ }^{*}$ Speaker. 


\section{Introduction}

Study of two- and three-nucleon short range correlations [1] affords us an opportunity to use the dense few-nucleon correlated systems of this type (SRC) as targets, which correspond to small fragments of nuclear matter in the dynamically broken chiral symmetry states. Collisions of SRC with bombarding particles can initiate the chiral phase transition ending in the creation of MB. Thus, its observation would be a direct evidence of the chiral condensate disappearance in the interaction area. Separation of a MB mass from the secondary particle background is feasible if the MB decay width is narrow enough. That requires excitation energy of produced MB to be low. For this purpose it is reasonable to select only those experimental events in which MB production is accompanied with a cumulative particle taking away an essential part of the excitation energy.

Prehistory of cumulative particle phenomena may be traced back to 1957, when some clear evidences for existence of simultaneous interactions of projectile proton with two close intranuclear nucleons was established by G.A. Leksin, M.G. Mescheryakov et al. and D.I. Blokhintsev [2 -4]. An important step was made by A.M. Baldin who considered such interactions in the frame of quark-parton model that does not add up to the description in terms of nucleons [5]. V.S. Stavinsky et al. found some experimental evidences for this point of view [6]. Further development of quark-parton approach was carried out by V.V. Burov, V.K. Lukianov and A.I. Titov who examined explicitly a possibility of occurrence of quark bags preexisting in nuclei before their interaction with the projectile particle [7-9]. They gave them a name of fluctons, though originally D.I. Blokhintsev called compact nucleon formations that name. More similar to the initial Blokhintsev's idea turned out to be the notion of SRC appeared nearly at the same time [10]. United explanation of the data on the cumulative particle production and the EMC-effect based on a suggestion of multiquark nature of fluctons was one more evidence for the flucton existence [11]. It is also worth noting a paper [12] in which an interesting parametrization of data on cumulative and subthreshold particle production was given. This made it possible to embed into one functional relation all experimental data known in this field. Such a scaling may also be considered as a hint about the parton structure of interacting objects.

\section{Method of cumulative $\pi$-meson and cumulative nucleon}

Let us consider a cumulative $\pi$-meson outgoing under angle $\theta$ with respect to momentum $\mathbf{p}_{0}$ of the projectile proton in the laboratory system. The law of conservation of energy-momentum gives:

$$
E_{0}+M=E_{\pi}+\left(p_{*}^{2}+M_{*}^{2}\right)^{1 / 2},
$$

where $M$ is a value of mass of SRC, undergoing the collision, $M_{*}$ is a mass of a total system in the final state aside from the cumulative meson, $\mathbf{p}_{*}$ is momentum of the system,

$$
p_{*}=\left(p_{\pi}^{2}-2 p_{\pi} p_{0} \cos \theta+p_{0}^{2}\right)^{1 / 2} .
$$

Relation (2.1) may be considered as a function $M_{*}(M)$ as far as all the values it contains are known from the experiment. We use $M_{*}(M)$ for estimation of the production possibility of $(n+1)$-baryon from $n$-nucleon SRC in the cumulative process of this type. As is known, SRC has a continuous 


\begin{tabular}{|l|c|c|c|}
\hline$P_{\pi}, \mathrm{GeV} / \mathrm{c}$ & $\mathrm{f}, \mathrm{Be}$ & $\mathrm{f}, \mathrm{Al}$ & $\mathrm{B}$ \\
\hline 0.873 & $1.6510^{-4}$ & $4.6110^{-4}$ & 2 \\
0.979 & $2.4710^{-5}$ & $8.6210^{-5}$ & 2,3 \\
1.077 & $3.7210^{-6}$ & $1.7210^{-5}$ & 2,3 \\
1.293 & $6.2310^{-8}$ & $3.5610^{-7}$ & 2,3 \\
1.402 & $8.2110^{-9}$ & $5.3210^{-8}$ & $2,3,4$ \\
1.512 & $7.9410^{-10}$ & $4.9510^{-9}$ & $2,3,4$ \\
1.619 & & $1.0310^{-9}$ & $2,3,4,5$ \\
\hline
\end{tabular}

Table 1: Upper bounds for the production cross section of MBs by $10.14 \mathrm{GeV}$ protons irradiating Be and Al. Cumulative pions are registered at laboratory angle $\theta=119^{\circ}$.

\begin{tabular}{|l|c|c|c|}
\hline$P_{\pi}, \mathrm{GeV} / \mathrm{c}$ & $\mathrm{f}, \mathrm{Be}$ & $\mathrm{f}, \mathrm{Al}$ & $\mathrm{B}$ \\
\hline 1.192 & $1.9510^{-5}$ & $7.0910^{-5}$ & 2 \\
1.370 & $1.2010^{-6}$ & $6.3410^{-6}$ & 2,3 \\
1.523 & $9.3610^{-8}$ & $6.3710^{-7}$ & 2,3 \\
1.635 & $1.4010^{-8}$ & $1.2610^{-7}$ & $2,3,4$ \\
1.790 & $1.2110^{-9}$ & $1.4210^{-8}$ & $2,3,4$ \\
\hline
\end{tabular}

Table 2: The same experiment as in Table 1 but $\theta=97^{\circ}$.

mass spectrum estimated at least for two-nucleon SRC. In particular, it was found that two-nucleon SRCs dominate the nuclear wave function at $k_{\min } \geq 300 \mathrm{MeV}$ [1]. Relying on this and taking into account an approximate proportionality of SRC masses to their baryon numbers, $n$, we accept for the minimal mass $m_{n}$ of n-nucleon SRC a value

$$
m_{n} \simeq n,
$$

where $n$ is taken in $\mathrm{GeV} / \mathrm{c}^{2}$ units. MB masses, $M_{n}$, are estimated here in the quark bag model framework [8]: $M_{3}=3.62, M_{4}=4.76, M_{5}=6.07 \mathrm{GeV} / \mathrm{c}^{2}$. A criterion of the possibility of the transition $n$-nucleon SRC to $(n+1)-\mathrm{MB}$ is chosen as

$$
M_{*}(M) \geq M_{n+1},
$$

and excitation energy of MB is $E_{e x}=\left(M_{*}(M)-M_{n+1}\right) c^{2}$.

A momentum of a cumulative meson accompanying the MB production decreases with decrease of an incident proton momentum. Thus, $p_{\pi}$ is equal only to $0.098 \mathrm{GeV} / \mathrm{c}$ for tribaryon production in $p+d$ interactions for $\theta=180^{\circ}$ and two-particle SRC mass $M=2.1 \mathrm{GeV}$. The reaction is aready impossible for $M=2.0 \mathrm{GeV}$. In Tables 1 and 2 results of calculations of the yield of MBs for the cumulative $\pi$-meson production from nuclear targets $\mathrm{Be}$ and $\mathrm{Al}$ for two angles $\theta=119^{\circ}$ and $\theta=97^{\circ}$ are shown. The first column encloses momenta of cumulative mesons, the fourth column contains baryon numbers of possible MBs. The experimental invariant cross sections, $f=A^{-1} E d \sigma / d^{3} p$, for cumulative meson production (in $\mathrm{mb} \cdot \mathrm{GeV}^{-2} \cdot \mathrm{c}^{3} \cdot \mathrm{sr}^{-1} \cdot$ nucleon ${ }^{-1}$ ) are shown in the second and third columns [13]. They are given for estimation of the feasibility of the 
measurements and represent an upper bound for the production cross section of corresponding $\mathrm{MB}$ (i.e. the production cross section of MB is less). One can see that values of $\theta$ and momentum of a cumulative $\pi$-meson should be as large as possible for observation of the chiral phase transition in fragments of nuclear matter big enough. This observation is in a qualitative agreement with a conclusion of [14] that the minimal number of interacting nucleons necessary for observation of a cumulative meson is

$$
N_{\min } \approx \frac{E_{\pi}-p_{\pi} \cos \theta}{m}
$$

where $m$ is the nucleon mass. Kinematics also permits us to determine the smallest value of cumulative meson momentum necessary for the MB production. Thus, one can find values $0.508,0.942$, 1.328 and $1.602 \mathrm{GeV} / \mathrm{c}$ corresponding to production of MBs with baryon numbers 2, 3, 4 and 5 shown in Table 1 and values $0.663,1.203,1.523,1.794 \mathrm{GeV} / \mathrm{c}$ for Table $2^{1}$.

Now it is appropriate to formulate a general strategy of the MB hunting:

- One should start from the minimal cumulative meson momentum corresponding to the minimal possible mass of the SRC, which is not far from the sum of masses of nucleons from which MB is composed. Since SRCs with the minimal mass value may be transformed into MB not so often, a chance to find MB in this cinematical region can be small for a while.

- Therefore, it is likely that one should proceed further and increase a value of momentum of the trigger cumulative meson. Now more energy is sent away from the region of reaction. But so long as a mass of MB is fixed, the interactions should start now with greater initial mass of SRC, and a higher density in it.

- Eventually, one should arrive to a value of $p_{\pi}$, where the MB may be seen as a narrow peak in the effective mass distribution. It is not advisable to go very far off the MB production threshold because of appearance of additional reaction channels in that region.

- The most convincing experiments for the MB production would be the following: $p+p \rightarrow$ dibaryon $+\pi, p+d \rightarrow$ tribaryon $+\pi$, and so on, in which a nucleus in the initial state is transformed as a whole into a final MB after interaction with the projectile proton. In such a case one avoids three main obstacles in the way of MB identification ${ }^{2}$ : a necessity to account for interactions with additional intranuclear nucleons, an ambiguity of total momentum of SRC inside a nucleus, and a need for identification of all particles constituting $X$ system in reactions of $p+A \rightarrow \pi+X$ type. A multibaryon with baryon number $B=1+A$ may be found as a peak in the invariant mass $M_{X}$ spectrum, where $M_{X}$ is calculated by making use the cumulative $\pi$-meson momentum only.

- It is also possible to observe transitions of a target nucleus into MB with baryon number $B=A$ for reactions $p+A \rightarrow p+\pi+Y$ with a sufficient proton momentum transfer as a peak in $M_{Y}$ mass distribution. In the general case, it will be optimal to scatter all experimental data over a plot $M_{Y}$ versus $M_{X}$. Regions of a high point density are possible indication of

\footnotetext{
${ }^{1}$ For estimation of the dibaryon production threshold we accepted for dibaryon mass a value $M_{2}=2.05 \mathrm{GeV} / \mathrm{c}$ experimentally observed in [15].

${ }^{2}$ Below we show formidability of these obstacles on the basis of the EVA spectrometer experiment.
} 
the MB production at those locations. A detection of the scattered proton in coincidence with a cumulative pion from the decay of an excited MB is required for measurement of $M_{Y}$, and only a detection of the cumulative pion together with some experimental cut imposed on secondary nucleon momenta, $p_{N}<p_{\text {cut }}$, is necessary for measurement of $M_{X}$. A value of $p_{c u t}$ is determined by a theoretical model of MB decay.

This strategy may be referred to as a method of cumulative meson. It is natural to verify it's efficiency for the case of dibaryon (2B) production in reactions

$$
p+H \rightarrow 2 B+\text { cumulative } \pi+\ldots
$$

and check correctness of theoretical models $[16,18]$ and experimental findings for dibaryon masses $[15,17,19,20]$. In the case of a sure identification at least one $2 B$, it would be useful to chose different targets to investigate formation of $2 \mathrm{~B}$ in $p+n$ system, A-dependence of $\pi$-meson intranuclear potential, etc.

Of course, the dibaryon formation still hardly resembles the chiral phase transition in a large nuclear volume. In the general case, research of the chiral phase transition in small fragments of nuclear matter calls for a careful analysis of finite size effects originated from the shell structure of a quark bag, the surface energy, and the Coulomb forces at short distances. Quark bag models predict nearly constant value of $M_{n} / n$ for $n=3,4,5$, corresponding to its bulk value (see, e.g., $[8,21,22])$. A slight, on 2-3\% accuracy level, deviation from this value is caused by the shell effects. In accordance with [21], surface tension coefficient for the quark bag is about $(70 \mathrm{MeV})^{3} \approx$ $8,8 \mathrm{MeV} / \mathrm{fm}^{2}$. This gives for $M_{3}$ a correction about $2-3 \%$ for the radius of the tribaryon $R \approx 0.8$ $\mathrm{fm}$ taken as for the flucton[8]. Independent consideration of the Casimir energy, which includes a contribution of surface tension energy, gives the same estimation within the bounds of the chiral bag model [23]. A compound $(\mathrm{n}+1)$-baryon system, consisting of the projectile proton and $n-$ baryon SRC, can acquire an additional mass increase due to the Coulomb repulsion of a charge of the projectile and a charge of SRC. This gives a correction to $M_{3}$ on $0.13 \%$ level or less. Thus, our estimations indicate that the finite size effects exert no essential influence upon the chiral phase transition in MBs with baryon numbers $B \geq 3$. Therefore, a detection of the tribaryon can be the simplest prototype of a discovery of the chiral phase transition which is now the object of search in heavy nucleus collisions.

Consideration of the cumulative nucleon is similar to the previous estimations for the pion. Results of calculations of yield of MBs for the cumulative proton production from nuclear targets Be and $\mathrm{Al}$ for two angles $\theta=119^{\circ}$ and $\theta=97^{\circ}$ are shown in Tables 3 and 4 . The smallest values of cumulative proton momentum necessary for the MB production for data shown in Table 3 are 0.964 , $1.415,1.834 \mathrm{GeV} / \mathrm{c}$ for $\mathrm{MBs}$ with $\mathrm{B}=2,3,4$. Corresponding values for Table 4 are 1.299, 1.827, $2.31 \mathrm{GeV} / \mathrm{c}$. One can see that using the cumulative nucleon is less effective than the employment of the cumulative pion. This is because of escape of nucleon from excited MB reduces a baryon number of residual MB. In addition, nucleon as a cooling agent is worse than pion, as far as it is heavier.

The most demonstrative experiments with cumulative particles correspond to the case in which a nucleus in the initial state is transformed as a whole into a final MB after absorption of the projectile proton. Concrete experimental proposals of this kind will be given below. But it is also 


\begin{tabular}{|l|c|c|c|}
\hline$P_{\pi}, \mathrm{GeV} / \mathrm{c}$ & $\mathrm{f}, \mathrm{Be}$ & $\mathrm{f}, \mathrm{Al}$ & $\mathrm{B}$ \\
\hline 0.874 & $1.2910^{-2}$ & $4.3910^{-2}$ & 1 \\
0.980 & $2.8910^{-3}$ & $1.1510^{-2}$ & 2 \\
1.402 & $3.2910^{-6}$ & $2.8510^{-5}$ & 2 \\
1.512 & $5.3910^{-7}$ & $5.0310^{-6}$ & 2,3 \\
1.738 & $8.0110^{-9}$ & $1.0810^{-7}$ & 2,3 \\
1.835 & $1.1010^{-9}$ & $2.2510^{-8}$ & $2,3,4$ \\
\hline
\end{tabular}

Table 3: An upper bounds for the production cross section of MBs by $10.14 \mathrm{GeV}$ protons irradiating nuclear targets of $\mathrm{Be}$ and $\mathrm{Al}$. The cumulative proton is registered at laboratory angle $\theta=119^{\circ}$.

\begin{tabular}{|l|c|c|c|}
\hline$P_{\pi}, \mathrm{GeV} / \mathrm{c}$ & $\mathrm{f}, \mathrm{Be}$ & $\mathrm{f}, \mathrm{Al}$ & $\mathrm{B}$ \\
\hline 1.192 & $1.5610^{-3}$ & $7.0210^{-3}$ & 1 \\
1.370 & $1.9510^{-4}$ & $1.0810^{-3}$ & 2 \\
1.790 & $4.2910^{-7}$ & $4.5310^{-6}$ & 2 \\
2.020 & $9.4910^{-9}$ & $1.5610^{-7}$ & 2,3 \\
\hline
\end{tabular}

Table 4: The same experiment as in Table 3 but $\theta=97^{\circ}$.

tempting to elaborate a theoretical model which allows us to deal with MB production from separate parts of nuclei, as far as cross sections of the processes under discussion are rather small. Such a model should include, particularly, an account of intranuclear scattering of secondary particles. As a certain step in that direction, we consider data taken with EVA spectrometer.

\section{Data taken with EVA spectrometer}

Reaction ${ }^{12} \mathrm{C}(p, 2 p+n)$ was studied the with EVA spectrometer at 5.9, 7.5, 8.0 and 9.0 GeV/c $[24,25]$, and an agreement with the SRC description was found in a scope of the quasi-elastic knockout model (QENM). QENM assumes that the projectile proton undergoes elastic scattering off a proton maintaining in a two-nucleon SRC and after that both of them escape from the nucleus without any interactions. The neutron, which was enclosed into SRC before scattering, becomes free too and escapes from the nucleus due to a high value of momentum it had in SRC. Thus, QENM neglects all interactions apart from one hard elastic p-p scattering. Of course, the validity of this approximation is disputable, and it is still unclear if the data tolerate other interpretations, or not.

QENM gives an expression for a proton momentum in SRC,

$$
\mathbf{p}_{f}=\mathbf{p}_{1}+\mathbf{p}_{2}-\mathbf{p}_{0}
$$

where $\mathbf{p}_{0}$ and $\mathbf{p}_{1}$ are momenta of the projectile proton before and after hard scattering, $\mathbf{p}_{2}$ is a momentum of intranuclear proton after hard scattering of the projectile. Neglecting an influence of intranuclear potential, QENM sets all these momenta equal to momenta of the incoming and observed protons. Subscript $f$ means that in the kinematics considered the intranuclear protons 
had momenta directed mainly forward (along $\mathrm{z}$ axis in an accepted notation). Thereafter, neutron momenta in SRC were turned mainly to the backward direction, $\mathbf{p}_{n} \approx-\mathbf{p}_{f}$. If the Fermi motion is neglected, the sign of the approximate equality may be replaced by the exact one. According to $[24,25]$, the Fermi motion corresponds to values of nucleon pair momenta along $\mathrm{z}$ direction $\left|p_{z}^{c m}\right| \leq 0.3 \mathrm{GeV} / \mathrm{c}$, as compared with momenta of the relative motion of nucleons $p_{z}^{r e l}=0.08 \div 0.52$ $\mathrm{GeV} / \mathrm{c}$ in SRC.

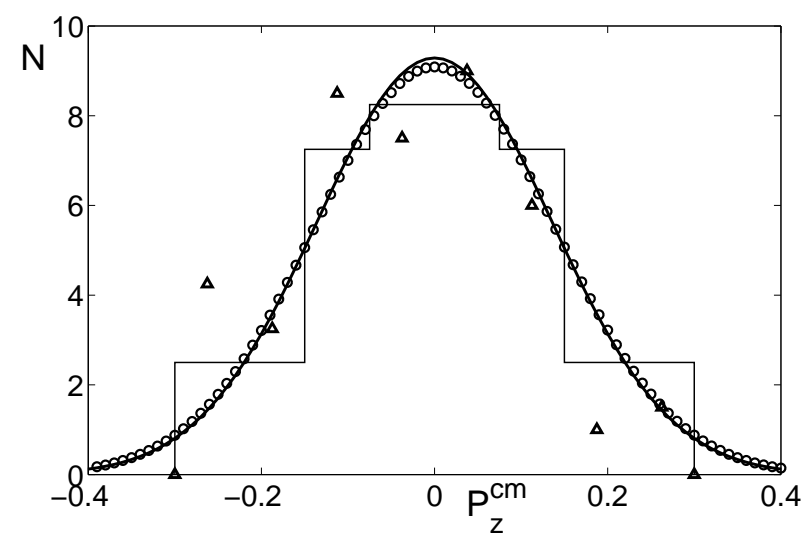

Figure 1: Comparison of theoretical and experimental distributions for z projection of the total Fermi momentum of the two nucleon system in ${ }^{12} \mathrm{C}$.

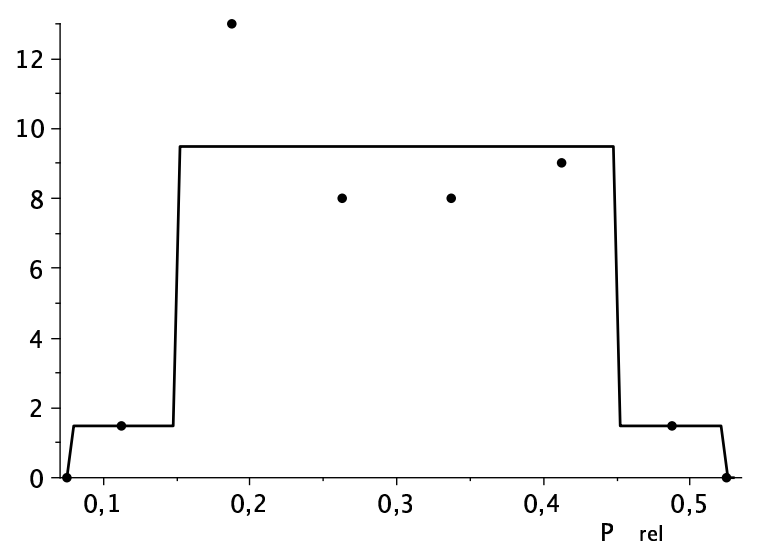

Figure 2: Experimental distributions for $\mathrm{z}$ projection of the relative momentum of nucleons in SRC (points) and smoothed data (histogram) used in our random simulation of SRC.

Experimental data for $p_{z}^{c m}$ are shown in Fig. 1 by triangles. Their averaged values (taking into consideration $p_{z} \rightarrow-p_{z}$ symmetry) are shown as the histogram. The line represents an approximation of the set of triangles by the Gaussian function $\left(\overline{p_{z}^{c m}}=0, \sigma=0.1358\right)$, and, for a comparison, open circles are the Gaussian approximation of the motion of pair in the ideal Fermi gas with $P_{F}=0.22 \mathrm{GeV} / \mathrm{c}$. One can see an unexpectedly good agreement between theory and experiment, which confirms the Fermi motion interpretation of $p_{z}^{c m}$ given in [24, 25].

Experimental data for $p_{z}^{\text {rel }}$ are shown in Fig. 2 by black points. As one can see, it is only possible to hope for recognition of mean value and dispersion of this distribution. For a smoothed 
curve shown in Fig. 2 as a histogram, they were found to be $\overline{p_{z}^{r e l}}=0.30, \sigma_{p_{z}^{r e l}}=0.098 \mathrm{GeV} / \mathrm{c}$.

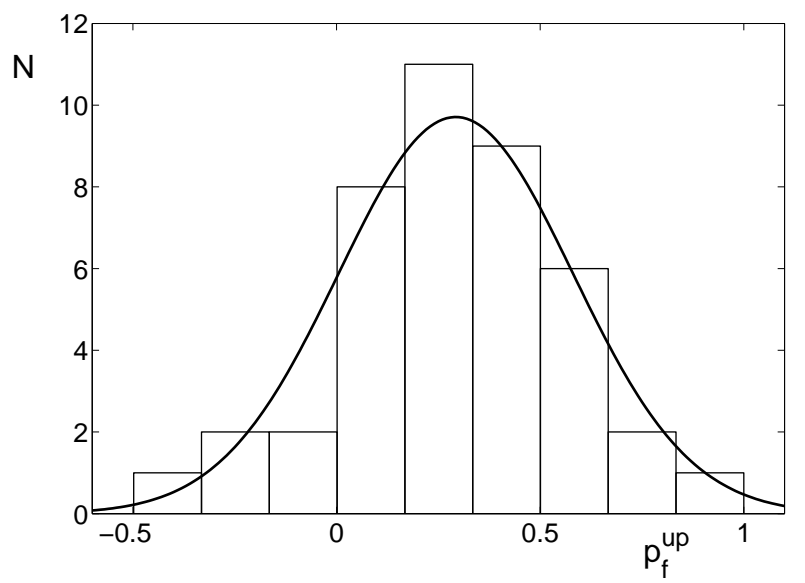

Figure 3: Comparison of the experimental distributions of $\mathrm{x}$ component of the proton momentum (histogram) with its theoretical approximation by a gaussoid.

For an estimation of momentum distributions of SRC nucleons in the transversal directions ( $x$ axis was chosen to be parallel to the upward direction in the laboratory), data for $p_{f}^{u p}$ shown in Fig. 1 in paper [25] have been used. It was established that the normal distribution with parameters $\overline{p_{f}^{u p}}=0.2928, \sigma_{f}^{u p}=0.2872 \mathrm{GeV} / \mathrm{c}^{\text {describes }}{ }^{3}$ the experimental data for different partitions of the registration region well enough, see, e.g., Fig. 3.

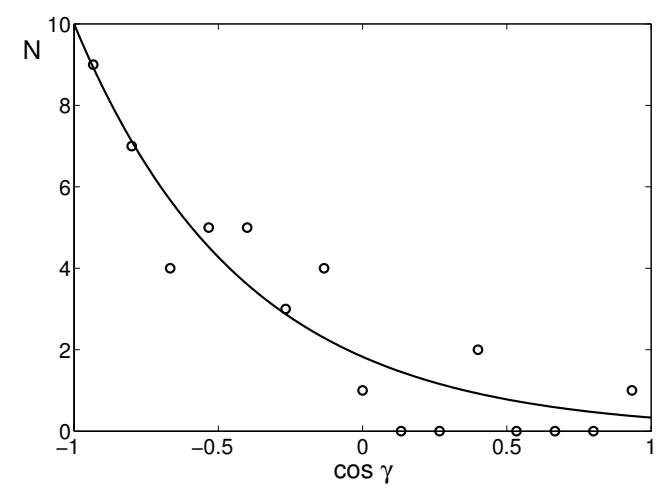

Figure 4: Experimental distribution for $\cos \gamma$ between momenta $\mathbf{p}_{n}$ and $\mathbf{p}_{f}$ (points) and its smoothing by an exponent $N=a \exp (-b(\cos \gamma+1)), a=10, b=1.7$ (line).

To establish the probability distributions of $\mathbf{p}^{c m}$ and $\mathbf{p}^{\text {rel }}$ in the transverse direction, let us consider two models:

M1. Probability distributions of $\mathbf{p}^{\mathrm{cm}}$ is suggested to be equal to that found for the longitudinal component, $\overline{p^{c m}}=0, \sigma=0.1358,-0.3<p^{c m}<0.3 \mathrm{GeV} / \mathrm{c}$. Relative motion is defined by a normal distribution with parameters: $\overline{p^{r e l}}=0.5856, \sigma=0.6,0<p^{r e l}<2$.

\footnotetext{
${ }^{3}$ Straightforward data averaging gives $\overline{p_{f}^{u p}}=0.35 \pm \delta, \sigma_{f}^{u p}=0.22$, where $\delta=\delta_{\text {stat }}+\delta_{\text {exp }}, \delta_{\text {stat }}=0.056$, and $\delta_{\text {exp }}=0.09$ is a mean value of the experimental error for $\overline{p_{f}^{u p}}$. Although values 0.2928 and 0.35 agree accurate within $\delta$, we prefer to assign values of parameters to the curve only after a rough recognition of its form, but not before it.
} 


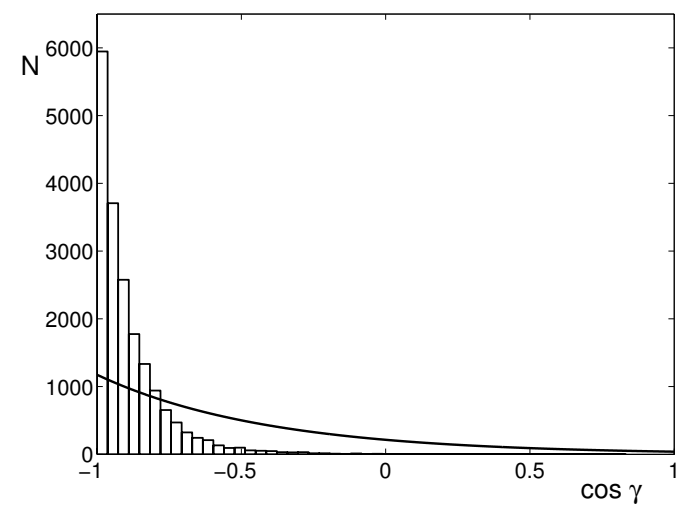

Figure 5: Description of the experimental data by M1.

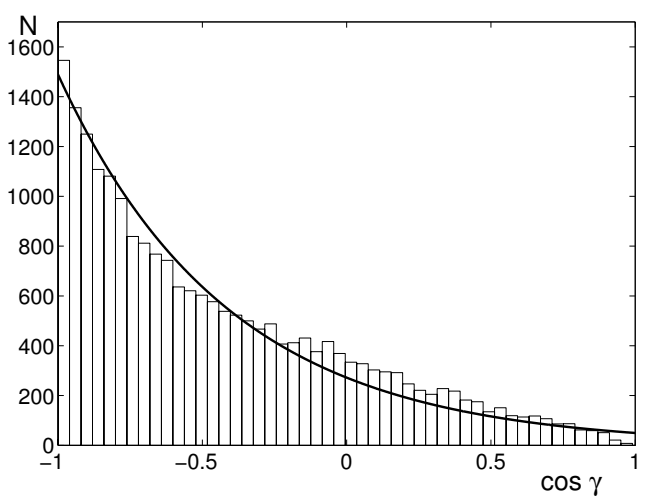

Figure 6: Description of the experimental data by M2.

M2. Distributions of $\mathbf{p}^{c m}$ is taken essentially wider. The total momentum of two nucleons is described by a normal distribution with parameters: $\overline{p^{c m}}=0, \sigma=0.57,-1.2<p^{c m}<1.2$. The relative motion is defined by a normal distribution with parameters: $\overline{p^{r e l}}=0.5856, \sigma=0.2121$, $0<p^{\text {rel }}<1.2$.

Model M1 is correct in the case when analysis of the experimental data based on QENM is true, and if the Fermi motion at the moment of hard scattering from ${ }^{12} \mathrm{C}$ is isotropic. Model M2 permits violations of those assumptions. It turns out that parameters of $\mathbf{p}^{r e l}$ can be chosen so that $p_{f}^{u p}$ distributions fit the experimental data extremely well for the both cases ${ }^{4}$.

To choose a proper model, the distribution for cosine of an angle $\gamma$ between neutron and proton momenta, $\mathbf{p}_{n}$ and $\mathbf{p}_{f}$, was calculated. An experimental distribution for $\cos \gamma$ is shown in Fig. 4 by points. Solid line corresponds to its exponential approximation found by the least square technique. Assuming identical distributions for $x$ and $y$ components of momenta, we found distributions of $\cos \gamma$ in the frameworks of M1 and M2, see Fig. 5 and 6. One can see that M2 is in a good agreement with experimental data, and M1 apparently overestimates asymmetry of the distribution

\footnotetext{
${ }^{4}$ An option $p_{f}^{u p} \geq 0$ was chosen to respect an approximate experimental cut $p_{f}^{u p} \geq 0$. Experimental events $p_{f}^{u p}<0$ taken into account for retrieving $p_{f}^{u p}$ distribution (see Fig. 3) are not considered hereafter.
} 
Our conclusion may be independently confirmed by the correlation analysis. Experimental values of $p_{n}$ and $p_{f}^{u p}$ in region $0.22<p_{n}<0.55, p_{f}^{u p}>0$ for pairs of secondary nucleons are shown in Fig. 7 . A quality of the model descriptions may be estimated by the instrumentality of linear regression analysis. One can write in the case under consideration:

$$
\left\langle p_{f}^{u p}\left(p_{n}\right)\right\rangle=\overline{p_{f}^{u p}}+\rho_{f n}^{\exp } \frac{\sigma_{f}^{u p}}{\sigma_{n}^{\exp }}\left(p_{n}-{\overline{p_{n}}}^{\exp }\right) .
$$

Parameters $\overline{p_{f}^{u p}}$ and $\sigma_{f}^{u p}$ entering into this relation were already discussed previously for the both experimental and theoretical straight lines. Degree of their match is determined by a quality of the previous experimental data approximation. A distinction of the two lines by height is just due to this factor (see footnote 3 above). An approximate agreement of experimental and simulated values of $\sigma_{n}$,

$$
\sigma_{n}^{\text {exp }}=0.086, \quad \sigma_{n}^{\text {sim }}=0.091
$$

may be considered as a confirmation of M2. Correlation coefficients are also matched quite good,

$$
\rho_{f n}^{s i m}=-0.19, \quad \rho_{f n}^{e x p}=-0.18
$$

without any special fitting.

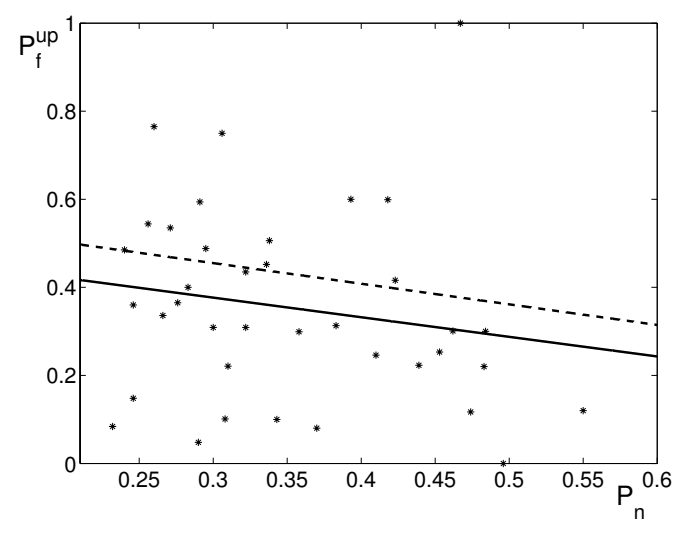

Figure 7: Relation between the component of proton momentum $p_{f}^{u p}$ and the total momentum of secondary neutron $p_{n}$. Points describes the experimental data [25], a straight solid line is their averaging found by the least-squares method. A dashed line corresponds to calculations in the frame of M2.

In Fig. 8, results of random simulation in M1 framework are shown as points. A dashed line describes $\left\langle p_{f}^{x}\left(p_{n}\right)\right\rangle$ dependence, a solid one was obtained in [24] on the basis of QENM. They coincide qualitatively and both of them give incorrect sign of the correlation coefficient. A distinction of biases for these two lines is explained by a poorer statistics accumulated at a moment when [24] was written. In spite of that, approximate accordance of the lines indicates that a model similar to M1 was kept in mind in [24]. Thus, our consideration demonstrates that the difference of the longitudinal and the transversal distributions for $p^{c m}$ escapes analyst's attention till now. Anticorrelation between values of the proton and neutron momenta obtained in the frame of M2 


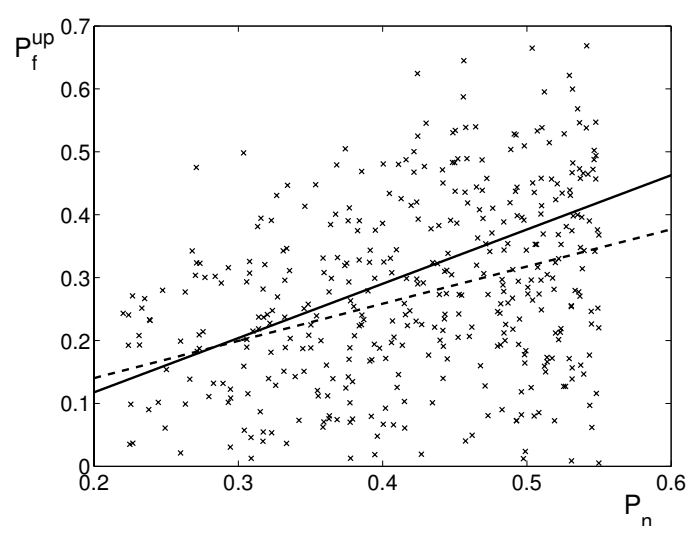

Figure 8: Dependence $\left\langle p_{f}^{x}\left(p_{n}\right)\right\rangle$ for the case when $p_{x}^{c m}$ and $p_{y}^{c m}$ distributions are described by M1.

may be interpreted as a strong influence of the center-of-mass motion on the SRC pair (the cmmotion acts upon nucleons in the opposite directions because of their reverse inner movement in SRC). The model M1 preserves sign plus of correlations between values of proton and neutron momenta, which takes place in the rest frame of SRC, due to a more moderate center-of-mass motion.

\section{A plausible cause for difference of longitudinal and transversal distributions}

It is clear that intrinsic restrictions of QENM are the basic cause of the impossibility to get a consistent description of the data, and now it is necessary to go beyond its scope. Let us designate momenta of the intranuclear proton and neutron, which enter into the SRC composition, by $\mathbf{p}_{p, f}$ and $\mathbf{p}_{n, b}$, correspondingly, and let $\mathbf{p}_{0}^{\prime}$ be a projectile proton momentum in a moment of its interaction with SRC. In the general case $\mathbf{p}_{0}^{\prime}=\mathbf{p}_{0}+\Delta \mathbf{p}_{0}$, where $\Delta \mathbf{p}_{0}$ is a momentum transferred to the projectile before its interaction with SRC. Similarly, let us write down momenta of nucleons measured by detectors in the following form: $\mathbf{p}_{i}^{\prime}=\mathbf{p}_{i}+\Delta \mathbf{p}_{i}$, where $\Delta \mathbf{p}_{i}$ is a momentum transfer gained by a nucleon with index $i$ on its way to the nuclear surface after SRC breakdown. Momentum conservation law for hard scattering by SRC in these notations takes a shape

$$
\mathbf{p}_{0}^{\prime}+\mathbf{p}_{p, f}+\mathbf{p}_{n, b}=\mathbf{p}_{1}+\mathbf{p}_{2}+\mathbf{p}_{n},
$$

where index $n$ denotes the neutron, and 1, 2 are proton indexes in the final state (we refer index 1 to the projectile proton scattered from SRC). It is proper to rewrite the last relation using only experimentally measurable momenta,

$$
\mathbf{p}_{0}+\mathbf{p}_{p, f}+\mathbf{p}_{n, b}=\mathbf{p}_{1}^{\prime}+\mathbf{p}_{2}^{\prime}+\mathbf{p}_{n}^{\prime}-\sum_{i} \Delta \mathbf{p}_{i}
$$

where index $i$ varies over $i=0,1,2, n$ values. In accordance with $[24,25]$ let us also define vectors

$$
\mathbf{p}_{f} \equiv \mathbf{p}_{1}^{\prime}+\mathbf{p}_{2}^{\prime}-\mathbf{p}_{0}, \quad \mathbf{p}^{c m} \equiv \mathbf{p}_{f}+\mathbf{p}_{n}^{\prime}, \quad \mathbf{p}^{r e l} \equiv \mathbf{p}_{f}-\mathbf{p}_{n}^{\prime}
$$


This implies the following expressions for vectors which are interpreted in scope of QENM as the total and relative momenta of nucleons constituting SRC,

$$
\begin{gathered}
\mathbf{p}^{c m}=\mathbf{p}_{p, f}+\mathbf{p}_{n, b}+\sum_{i} \Delta \mathbf{p}_{i}, \\
\mathbf{p}^{r e l}=\mathbf{p}_{p, f}+\mathbf{p}_{n, b}-2 \mathbf{p}_{n}^{\prime}+\sum_{i} \Delta \mathbf{p}_{i} .
\end{gathered}
$$

Formula (4.1) allows, at least in principle, to explain the difference of longitudinal and transversal distributions for $p^{c m}$ by a more sufficient contribution of intranuclear scattering into the transversal momentum. Indeed, since particles 0 and 1 have much higher momenta than others, and these momenta are directed mainly (or totally) along the longitudinal direction, their elastic scattering should be characterized by a large momentum transfer just in the transversal direction:

$$
\left|\Delta p_{0}^{x}\right| \gg\left|\Delta p_{0}^{z}\right|, \quad\left|\Delta p_{1}^{x}\right| \gg\left|\Delta p_{1}^{z}\right| .
$$

\section{Simulation of scattering particles 0 and 1}

Experimental data [27] for elastic nucleon-nucleon scattering, $4.5<P_{0}<11 \mathrm{GeV} / \mathrm{c}$, approximated by formula

$$
\frac{d \sigma}{d|t|}=A \exp (B t)
$$

were used for description of interaction of the projectile proton before and after hard scattering off SRC see Fig. 9 and Fig. 10. A difference between domains of $t$ in the figures is for lack of ex-

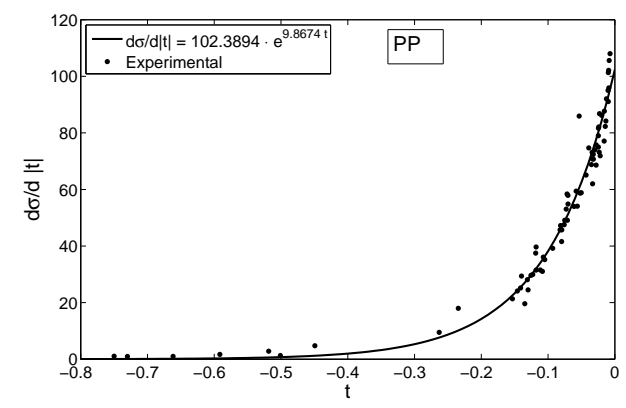

Figure 9: Experimental data for elastic proton-proton scattering at small angles, approximated by exponent.

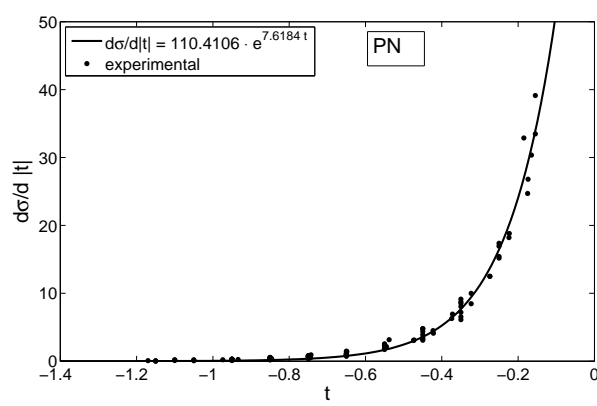

Figure 10: Experimental data for elastic proton-neutron scattering at small angles, approximated by exponent.

perimental data on elastic proton-neutron scattering at small angles. We also considered an impact of $t$ - and $s$-dependence of the coefficients $\mathrm{A}$ and $\mathrm{B}$ on the description of scattering. Experimental data for greater values of $|t|$ are shown in Fig. 11. Blue points correspond to $4.5<P_{0}<7.5 \mathrm{GeV} / \mathrm{c}$, red ones are for $7.5<P_{0}<11 \mathrm{GeV} / \mathrm{c}$. Solid line is approximation of the data by exponent with parameters $A=99.1, B=8.16 \mathrm{GeV} / \mathrm{c}$. It was chosen to be more close to the blue points since major portion of experimental data was taken at $P_{0}=5.9 \mathrm{GeV} / \mathrm{c}$. Our conclusions turn out to be 


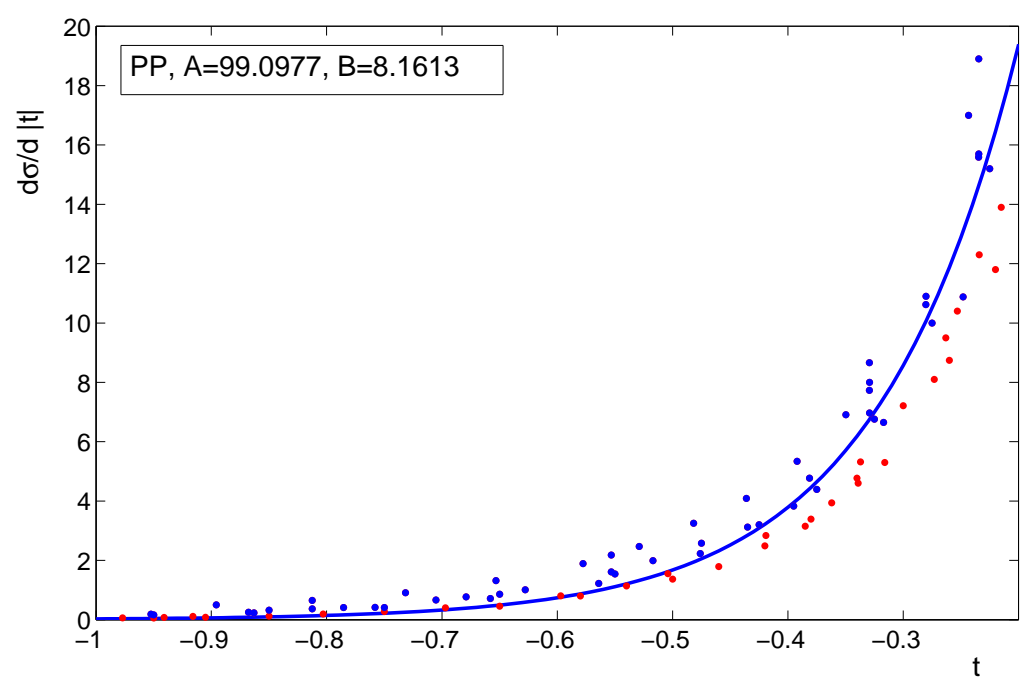

Figure 11: Experimental data for elastic proton-proton scattering at greater angles, approximated by exponent.

independent on a choice of approximation, and in what follows we use data shown in Fig. 9 and Fig. 10.

Let us specify main suggestions used in a program simulating elastic scattering the projectile before and after collision with SRC.

- Nuclear density $\rho$ is chosen in the Woods-Sakson form.

- Radius of nucleon core is $r_{N}=0.2 \mathrm{fm}$, binding energy of nucleons in ${ }^{12} \mathrm{C}$ is $6 \mathrm{MeV}$.

- Probability to find 2-nucleon SRC is proportional to $\rho^{2}$.

- The Pauli principle was respected, the Fermi momentum of nucleons in ${ }^{12} \mathrm{C}$ is $0.22 \mathrm{GeV} / \mathrm{c}$.

- There were no $\pi$-mesons accompanying secondary nucleons in the final state.

- Only elastic scattering the projectile before and after collisions with SRC is taken into account.

Distributions of number of interactions of particles 0 and 1 are shown in Fig. 12. The calculations revealed projectile proton does not experience elastic scattering in 87 percent of cases before hard scattering from SRC and in 74 percent of cases after it.

A contribution of intranuclear scattering of particles 0 and 1 into transversal momenta is shown in Fig. 13 and Fig. 14. It is interesting to note that both of the distributions have nearly the same width, though at first sight the second one should be noticeably wider. That is because a value of probability for simultaneous scattering of particles 0 and 1 is very small, $P=0.035$. Points in Fig. 13 and 14 describe the results of simulation, a solid line corresponds to their approximation by the Gauss distribution. Suppression of the distributions at small $p_{x}$ is caused by Pauli's principle. 


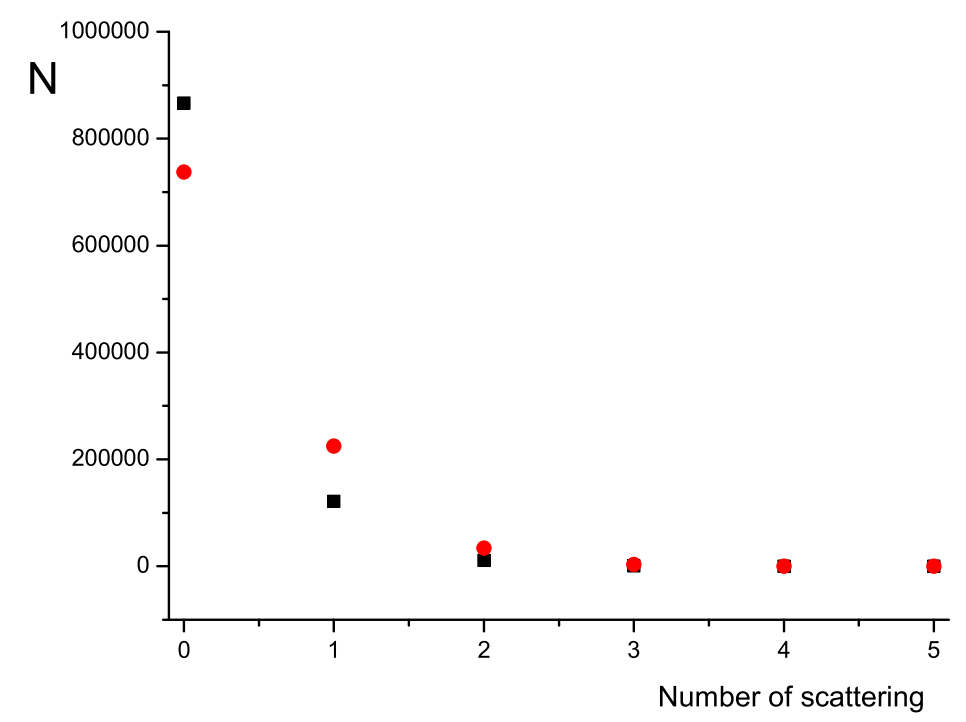

Figure 12: Distributions for number of elastic scattering projectile proton before and after interactions with SRC (black square boxes and red bubbles, correspondingly).

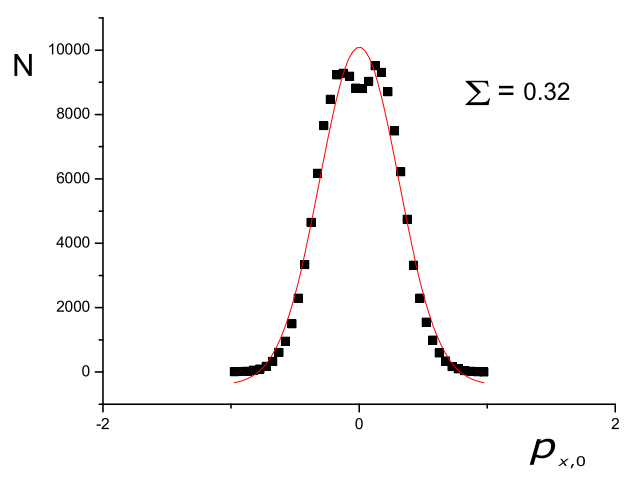

Figure 13: Distribution of an additional transversal momentum gained along $x$ axis as a result of scattering particle 0 .

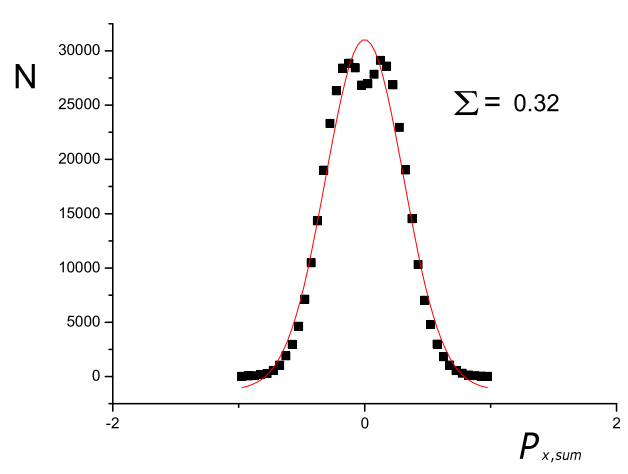

Figure 14: Distribution of an additional transversal momentum gained along $x$ axis as a result of scattering particles 0 and 1 .

Now it is possible to inquire whether scattering particles 0 and 1 may indeed be responsible for the difference between $p_{z}^{c m}$ and $p_{x}^{c m}$ distributions. Let us consider a random value $\xi$ which is the algebraic sum of two others,

$$
\xi=\xi_{1}+\xi_{2}
$$

which have zero mean values, $\overline{\xi_{1}}=\overline{\xi_{2}}=0$. In the general case one can write for the standard 
deviation of $\xi$

$$
\sigma_{\xi}^{2}=\sigma_{1}^{2}+\sigma_{2}^{2}+2 \rho_{12} \sigma_{1} \sigma_{2}
$$

where $\rho_{12}$ is the correlation coefficient between random values $\xi_{1}$ and $\xi_{1}$, and $\sigma_{1}$ and $\sigma_{2}$ are their standard deviations. For the present instance $\xi_{1}$ and $\xi_{1}$ correspond to $x$ components of vectors $\mathbf{p}_{p, f}+\mathbf{p}_{n, b}$ and $\sum_{i} \Delta \mathbf{p}_{i}$, accordingly, in the right-hand part of (4.1), and $\rho_{12}=0, \sigma_{1}=0.1358 \mathrm{GeV} / \mathrm{c}$. Here we took into consideration independence of the Fermi motion of SRC and scattering particles 0 and 1 , as well as Fig. 1 . The random value $\xi_{2}$ is dichotomous one. It is equal to zero with probability 0.64 , otherwise it is the random value shown in Fig. 14. Therefore,

$$
\sigma_{\xi}^{2}=\sigma_{1}^{2}+0.36 \Sigma^{2}=0.0553 \quad \Rightarrow \quad \sigma_{\xi}=0.235 .
$$

This value is appreciably less than the value 0.57 obtained in scope of M2. Moreover, it would be impossible to reproduce the value 0.57 even in the occurrence each event being accompanied by scattering particles 0 or 1 , since one has $\sigma_{\xi}=0.348 \mathrm{GeV} / \mathrm{c}$ in the latter case.

\section{Other possible explanations}

One more possibility should be checked first of all is a suggestion that there were $\pi$-mesons missed in experiments $[24,25]$. Indeed, the term $\sum_{i} \Delta \mathbf{p}_{i}$ in the right-hand part of (4.1) gives a more considerable contribution in the case when the $\pi$-meson production takes place, so that dispersion of $\mathbf{p}^{c m}$ calculated according to (4.1) becomes greater. However, it is still unclear if an essential meson production may be reconciled with a good description of $p_{z}^{c m}$ shown in Fig. 1, because those processes should be accompanied too with considerable momentum transfers along the longitudinal direction.

Logic permits also an assertion antipodal to the color transparency (an enhancement of the proton ability to interact with intranuclear matter after hard scattering). Such a suggestion is possible, at least in principle, because of an uncertain status of the color transparency for nucleons at the present time [28]. But since this suggestion obviously contradicts other experimental data obtained at BNL [29], it is more doubtful than the previous one.

An explanation which is based on neither experimental nor theoretical ambiguities may be as follows. Let us suppose that wave vector of ${ }^{12} \mathrm{C}$ has an admixture of states of a type:

$$
|\psi\rangle=\ldots+\alpha\left|\psi_{\perp}\right\rangle \otimes\left|\psi_{\|}\right\rangle,
$$

where $\left|\psi_{\perp}\right\rangle$ and $\left|\psi_{\|}\right\rangle$describe the transversal and longitudinal degrees of freedom. In such a case measurements of the longitudinal and transversal dimensions of nucleus may be fulfilled independently and may give different results. In connection with the EVA experiments, it is possible to suggest that the selection of events with a large momentum transfer along the transversal direction was equivalent to a selection of nuclear states more compact (squeezed) in that direction. Therefore, the Fermi motion of SRC measured along the transversal direction turned out to be more intense than it was along the longitudinal one. An experimental selection of this type is possible in the case of short range correlation arising only in the squeezed nuclear states.

In fact, none of the considered assumptions conflicts with another. For example, the $\pi$-meson production may be preceded by an appearance of a "large-sized" baryon strongly interacting with nuclear matter. 


\section{What has been observed in the EVA spectrometer experiment?}

Now it is natural to ask if the experimental data $[24,25]$ may be definitely interpreted as an evidence for SRCs? To this end a program simulating ${ }^{12} \mathrm{C}(p, 2 p+n)$ reactions was elaborated. Momentum-space wave function of SRC was chosen in the form of high momentum deuteron $S-$ and $D$ - state wave functions, see solid lines in Fig.2 in [26]. The quark counting rules [30, 31] were taken into account ${ }^{5}$ when sampling momenta of nucleons in SRC. Results of calculations are shown in Fig. 15 simultaneously with the experimental data, where the experiment corresponds to a more narrow curve. A theoretical curve would be even much wider provided experimental cuts were not rigorously simulated. In spite of an evident discrepancy between the curves, the probability of its occurrence is rather high, $p=0.84$, due to a small number of experimental events accumulated in $[24,25]$. It is possible to clarify this ambiguity detecting neutrons with higher momenta, or increasing statistics.

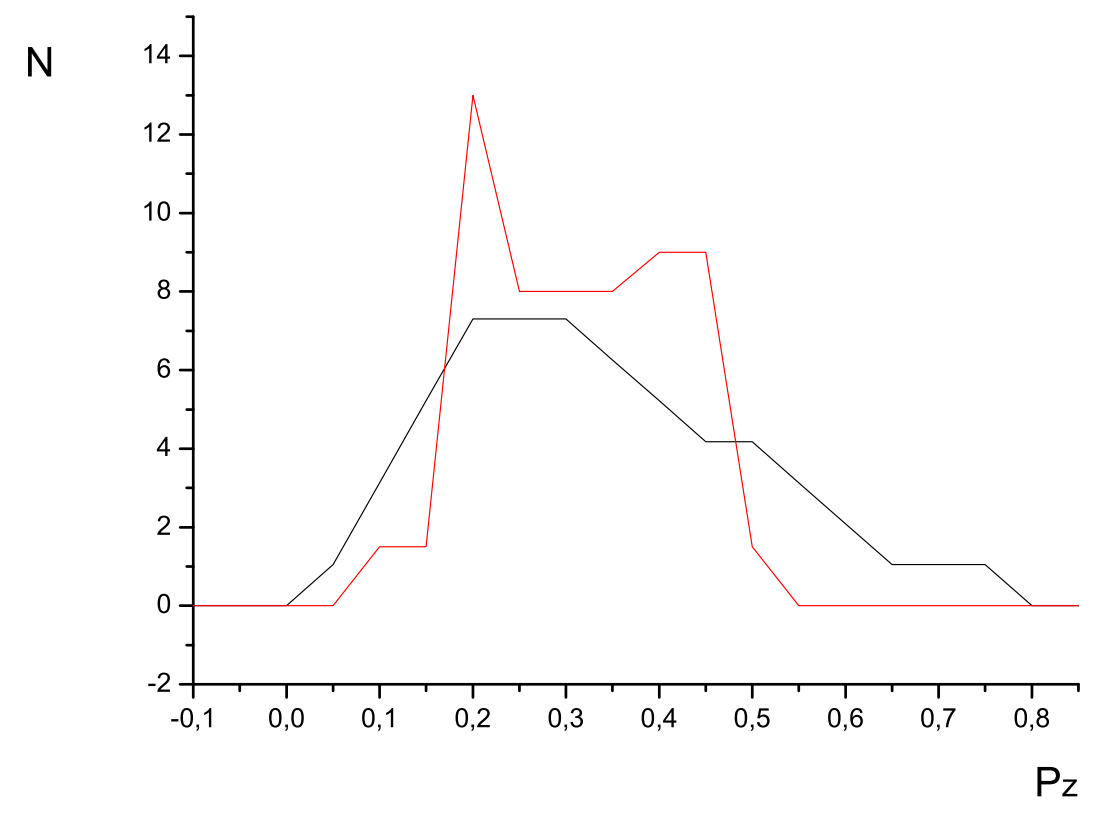

Figure 15: Comparison of theoretical and experimental distributions for the longitudinal component of the relative nucleon momentum in two nucleon SRCs (black and red curves, accordingly). The latter distribution is the same as shown in Fig. 2.

We also check plausibility of existence of the quark bags before or after hard scattering (fluctons and MBs, correspondingly). Distribution of the effective mass of nucleon pairs corresponding two nucleon SRC found in the M2 framework is shown in Fig. 16. Its peak belongs to an interval of possible values of 2-flucton masses ( $2 m_{N}$ plus $\left.0.2-0.3 \mathrm{GeV} / \mathrm{c}\right)$ estimated in [9]. Observed

\footnotetext{
${ }^{5}$ Because of them hard scattering off nucleons "running away" from the projectile particle is more probable than off nucleons "running toward".
} 
experimental dibaryon masses should lie in the vicinity of the curve maximum, but they were not distinguished because of an insufficient array of the experimental data given in [24, 25].

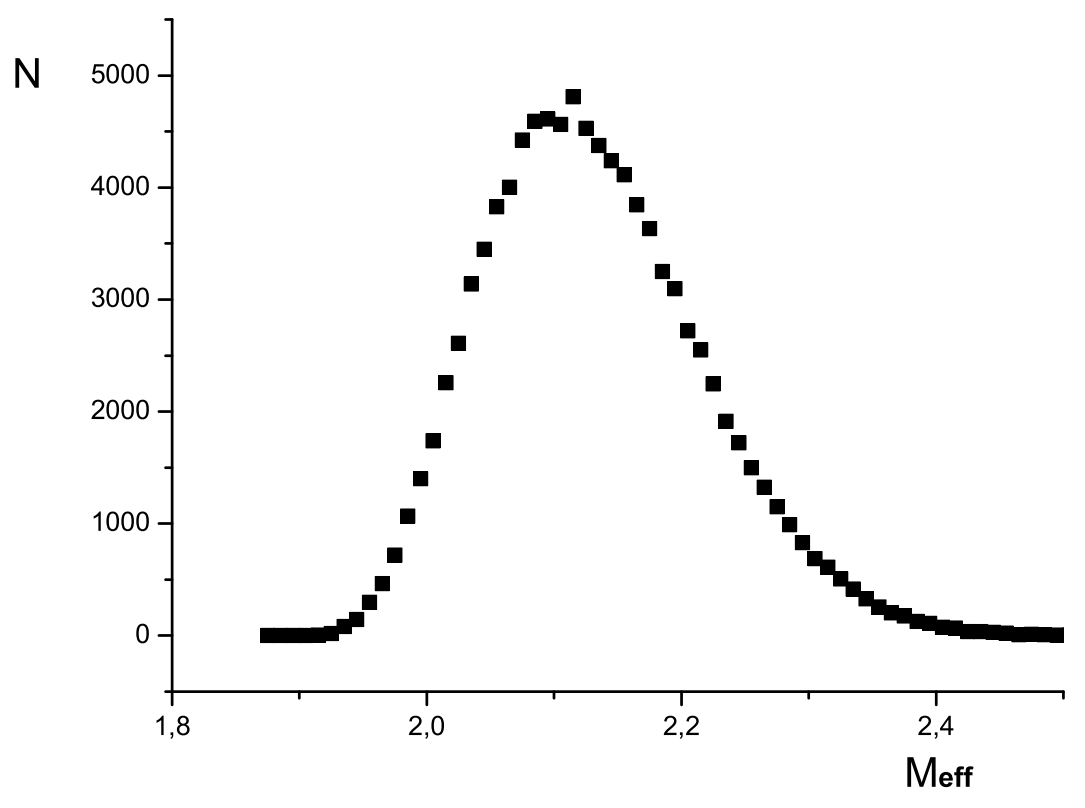

Figure 16: Experimental dibaryon masses have been observed at 1.950, 2.050, 2.122, 2.150 [15, 34], $1.955 \pm 2,1.965,1.980,1.999,2.008,2.017,2.046,2.087,2.106,2.129,2.172,2.238,2.283$ [17], 1.95, $2.02,2.12,2.18 \mathrm{GeV} / \mathrm{c}^{2}[35]$.

\section{Conclusion}

At present a possible approach to investigation of chiral phase transitions in few-nucleon system consists seemingly in study of interactions of accelerated protons with light nuclei with a cumulative meson in the final state. Theoretical description and experimental identification of MBs are not confronted with insuperable difficulties only in the case when all nucleons in the target nucleus are in the SRC state. One state of this kind has been already observed for ${ }^{3} \mathrm{He}$ in [1]. Therefore, it is reasonable to start study with $\mathrm{p}+{ }^{3} \mathrm{He} \rightarrow \mathrm{p}+\pi+3 \mathrm{~B}, \mathrm{p}+{ }^{3} \mathrm{He} \rightarrow \pi+4 \mathrm{~B}$ reactions. The main experimental problem to be solved consists in small cross sections of the processes. Its resolution requires an employment of accelerators with high-luminosity at a few GeV/c of the proton momentum. Kinematics allows to separate events with MB in the final state near its producing threshold using the cumulative meson as a trigger. One can estimate an upper bound for MB production cross sections using, e.g., data in Tables 1 and 2 for Be nucleus and a relation $A=2.528+0.468 N_{3}+0.004 N_{3}^{2}$ between number $N_{3}$ of three nucleon SRC and atomic number for light nuclei, $3 \leq A \leq 12$ (an approximation of the data [1]). The main theoretical problem consists in lack of knowledge about the wave function of 3N SRC, which is necessary for 3B and 4B identification. It may be obtained in experimental studies of ${ }^{3} \mathrm{He}\left(e, e^{\prime} \mathrm{N}\right) \mathrm{NN}$ reactions, which theoretical description was considered in [32]. 
We have not succeeded in deciding a question whether the EVA spectrometer experiment [24, 25] bears evidence for existence of SRCs only, or else for fluctons. It would be possible to cast light upon this problem using hard $\mathrm{p}+{ }^{3} \mathrm{He}$ hard scattering without a cumulative meson in the final state to knock out 3B fluctons from helium. The difference between flucton and SRC knockingout is quite distinctive, because in the latter case momentum distributions for secondary particles should be wider. A potential signal of this may be already seen in Fig. 15. It would be best of all to select only events without $\pi$-mesons in the final state, although for flucton detection they may be seemingly allowed. One may verify that none of the secondary particles was missed calculating their effective mass, which is also can be found using momentum transferred to the impinging proton. Quark bag nocking-out and its production may be distinguished too because the former corresponds to elastic scattering without additional energy loss spent on the MB production.

It worth noting that tribaryon production might already manifest itself in nuclear reactions, but was not recognized due to lack of a special attention to it. For example, cross section of reaction $D+D \rightarrow X+D$ at $8.9 \mathrm{GeV} / \mathrm{c}$ momentum of primary deuterons and $t=-0.5(\mathrm{GeV} / \mathrm{c})^{2}$ was measured in [33]. At those conditions, different angles of recoil deuterons correspond to different values of $M_{X}$. Three peaks were clearly observed in $M_{X}$ dependence of the cross section: at $M_{X}=$ $M_{D}, M_{X} \approx M_{D}+0.07 \mathrm{GeV} / \mathrm{c}^{2}$ and $M_{X} \approx M_{D}+0.46 \mathrm{GeV} / \mathrm{c}^{2}$. The first peak may be identified with elastic D-D scattering, the second one corresponds to a narrow dibaryon resonance $M_{2 B}=$ $1.95 \mathrm{GeV} / \mathrm{c}^{2}$ observed lately in three independent experiments $[17,34,35]$, though there were no comments on this subject in [33]. The third peak, rather wide, may arise due to intermediate tribaryon appearance, $D+D \rightarrow 3 B+N \rightarrow X+D$, at $M_{3 B} \approx 3.28 \mathrm{GeV} / \mathrm{c}^{2}$.

One may suggest that synthesis of multibaryons will be considered in the future as a crucial issue, taking into account its significance for QCD. Bearing that in mind, we made a list of names for multibaryons with baryon numbers from 1 to 10 using designations generally accepted in mathematics and chemistry:

- $1 \mathrm{~B}=$ monobaryon

- $2 \mathrm{~B}=$ dibaryon

- $3 \mathrm{~B}=$ tribaryon

- $4 \mathrm{~B}=$ tetrabaryon

- $5 \mathrm{~B}=$ pentabaryon

- $6 \mathrm{~B}=$ hexabaryon

- $7 \mathrm{~B}=$ heptabaryon

- $8 \mathrm{~B}=$ octabaryon

- $9 \mathrm{~B}=$ nonabaryon

- $10 \mathrm{~B}=$ decabaryon 


\section{References}

[1] K.S. Egiyan et al. (CLAS Collaboration), Phys. Rev. Lett. 96 (2006) 082501.

[2] G.A. Leksin, JETP 32 (1957) 445.

[3] L.S. Azhgirey et al., JETP 33 (1957) 1185.

[4] D.I. Blokhintsev, JETP 33 (1957) 1295.

[5] A.M. Baldin, Bull. Lebedev Phys. Institute 18 (1971) 465.

[6] A.M. Baldin et al., Yad. Fiz. 21 (1975) 1008.

[7] V.V. Burov, V.K. Lukianov, A.I. Titov, Phys. Lett. B 67 (1977) 46.

[8] V.K. Lukianov, A.I. Titov, PEPAN 10 (1979) 815.

[9] V.V. Burov, V.K. Lukianov, A.I. Titov, PEPAN 15 (1984) 1249.

[10] L.L. Frankfurt, M.I. Strikman, PEPAN 11 (1980) 571.

[11] A.V. Efremov, Nuclear Structure Functions and Cumulative Processes (1997) [hep-ph/9710411v1].

[12] A.A. Baldin, Phys. Atom. Nucl. 56 (1993) 385.

[13] S.V.Boyarinov et al., Yad. Fiz. 46 (1987) 1472; 57 (1994) 1452.

[14] V.S. Stavinsky, PEPAN 10 (1979) 949.

[15] B. Tatischeff et al., Phys. Rev. C 59 (1999) 1878.

[16] S.M. Dorkin, B.L. Reznik, A.I. Titov, Yad. Fiz. 36 (1982) 1244.

[17] Yu.A. Troyan, PEPAN 24 (1993) 683.

[18] P.J. Mulders, A.T. Aerts, J.J. de Swart, Phys. Rev. D 21 (1980) 2653.

[19] L.V. Fil'kov et al., Baldin ISHEPP XV (2000) [nucl-th/0101021v2].

[20] M.M. Makarov, Physics-Uspekhi, 25 (1982) 83.

[21] E. Farhi, R.L. Jaffe, Phys. Rev. D 30 (1984) 2379.

[22] J.J. de Swart, Phys. Rev. D 17 (1978) 260.

[23] L. Vepstas, A.D. Jackson, Phys. Rep. 187 (1990) 109.

[24] J. Aclander et al., Phys. Lett. B 453 (1999) 211.

[25] A. Tang et al., Phys. Rev. Lett. 90 (2003) 042301.

[26] R. Gilman, F. Gross, J. Phys. G: Nucl. Part. Phys. 28 (2002) R37-R116.

[27] Landolt-Bornstein Numerical Data and Functional Relationships in Science and Technology, Group I: Nuclear and Particle Physics, V. 7, Springer-Verlag, Berlin-Heidelberg-New York 1973.

[28] G.A. Miller, Color Transparency (2012) [arXiv:1208.36668v1].

[29] J. Aclander et al., Phys. Rev. C 70 (2004) 015208.

[30] V.A. Matveev, R.M. Muradian, A.N. Tavkhelidze, Lett. Nuovo Cimento 7 (1973) 719.

[31] S.J. Brodsky, G.R. Farrar, Phys. Rev. Lett. 31 (1973) 1153.

[32] M.M. Sargsian et al., Phys. Rev. C 71 (2005) 044615. 
[33] A.M. Baldin et al., Differential Elastic Proton-Proton, Nucleon-Deuteron and Deuteron-Deuteron Scattering at Big Transfer Momenta (1979) JINR Communication 1-12397.

[34] B. Tatischeff et al., Phys. Rev. C 45 (1992) 2005.

[35] A. Deloff, T. Siemiarczuk, Z. Phys. A 555 (1993) 659. 